\title{
Insights of Pharmaceutical Co crystals
}

\author{
Preshita P Desai* \\ Department of Pharmaceutical Sciences, College of Pharmacy, Western University of Health Sciences, USA
}

Received: March 13, 2018; Published: March 29, 2018

*Corresponding author: FPreshita P Desai, Department of Pharmaceutical Sciences, College of Pharmacy, Western University of Health Sciences, Pomona 91766, California, USA, Tel: +1 909-469-6475; Email: preshita1712@gmail.com

Abbreviations: API: Active pharmaceutical ingredients, BCS: Biopharmaceutical Classification System; USFDA: United States Food and Drug Administration; EMA: European Medical Agency

\section{Introduction}

Active pharmaceutical ingredients (API) and API drug products developments are facing impending challenges as approximately 60-70\% of newly discovered APIs belong to biopharmaceutical classification system (BCS) Class II (poor solubility, good permeability) or BCS Class IV (poor solubility, poor permeability) which results in low oral bioavailability [1]. The conventional approaches of formulating API salts and amorphous APIs are also limiting because in most of these cases APIs either lack ionizable functionalities or do not have strong ionization to allow salt formation. Also, solubility enhanced amorphous form of APIs are thermodynamically unstable and are prone to transformation in more stable but less soluble crystalline form upon storage. Further, $\mathrm{pH}$ gradient across gastro intestinal 5track adds an additional challenge as some of these APIs possess variable $\mathrm{pH}$ dependent solubility and some are unstable or are prone to degradation. In this context, alternative approaches are being researched extensively.

Among these, advent of co crystals for pharmaceutical applications has gained wide attention over the past decade and co crystal engineering is being looked upon as a promising alternative to overcome poor solubility and bioavailability challenges. Briefly, pharmaceutical co crystals can be defined as one-phase crystal lattice comprising of a specific stoichiometry ratio of an API and a conformer which may or may not be pharmacologically active [2]. Notably, co crystals are nonionic supramolecular crystal lattice of the API and conformer which distinguishes them from salts, solvates, hydrates and polymorphs. The unique advantage of co crystals is the very fact that they not only retain the pharmacological activity of the API but also allow engineering of the physicochemical properties by virtue of the conformer and newly formed crystal lattice. Therefore, it can be surmised that with meticulous selection of co former, the API co crystal structure can be tailored to offer desired properties like superior solubility, permeability, stability, bioavailability, suitable melting point, particle size and morphology, flow properties etc.

\section{Feasibility and Approaches}

The most challenging aspect of co crystal engineering is selection of appropriate co former. A detailed scientific understanding of co former properties, interactions between API and co former, and its impact on properties of co crystal lattice are the key steps in this development. In early days, dibasic acids, nicotinamide etc. were used at large as co formers but researchers are now shifting focus towards more specialized co formers to achieve specific co crystal properties. In silico approaches enabling understanding of an API-co former interaction and prediction of the possible 3-D structure of co crystal lattice are coming on forefront as a preliminary investigation tools for this purpose. Typically, in the co crystal lattice, the non-ionic interactions between the API and co former majorly comprise of hydrogen bonding and other possible interactions like Vander Waal forces, hydrophobic interactions etc.

One must consider these critically while selecting suitable co former and for this, in silico molecular interaction software's, Hildebrand's and Hansen's solubility parameters, hydrogen bond donor acceptor analysis etc. can be used at an advantage. Additionally, if the objective is to enhance the solubility, the focus should be driven on developing a crystal lattice with reduced surface energy. Literature reports variety of methods to produce co crystals which can be grouped under two broad classes of solvent assisted methods (controlled solvent removal, supercritical solvent and antisolvent methods, sublimation etc.) and high shear methods (co-grinding, high shear mixing using planetary/ ball mill, kneading etc.). Selection of appropriate method should be rationalized based on the physicochemical properties of API and co former viz. melting point, heat stability, solvent solubility and stability etc.; the energy 
required to enable the API and co former interaction to form the co crystal; co crystal recovery and scalability [2].

\section{Regulatory Environment}

The regulatory agencies perspective towards co crystals is very crucial from clinical and commercialization aspect. To clarify this, United States Food and Drug Administration (USFDA) recently published industry guidance in February 2018 defining the regulatory classification for pharmaceutical co crystals. To confirm the API co crystal form, the guideline demands clear and in-depth screening to confirm that

a) API and co former concurrently exist in the crystal unit cell;

b) API and co former interact with non-ionic interactions only (differentiate them from salts, polymorphs, solvates and hydrates);

c) Significant dissociation of API from the co crystal prior to reaching the pharmacological site of action, the additional point of concern is if a co crystal should be considered as a pure physical mixture or a new chemical entity. It also becomes a complicated question when a co crystal comprises of two or more APIs. In response to this, USFDA clearly defines co crystal as a pure physical form and not a new chemical entity. It classifies co crystal analogous to the polymorph of API. Further, it describes that the co crystal comprising two or more APIs should be reported as fixed dose combination of those APIs. It is recommended that pharmaceutical investigators working in this area should critically consider these guidelines while developing and characterizing the API co crystals [3]. European Medical Agency (EMA) released a paper describing pharmaceutical use of co crystals but has not released specific regulatory guidelines till date $[4,5]$. Thought this gives a roadmap for co crystal application, the uncertainty in exact regulatory guidance is expected to make the co crystal based product approvals in this region comparatively tricky and challenging.

\section{Scope and Future Prospects}

Currently, very few co crystal based products have made their entry in the market. For example Entresto $₫$ (valsartan-sacubitril co crystal approved for management of heart failure), Lexapro® (escitalopram oxalate co crystal approved for management of anxiety disorders), some are under clinical investigation like ongoing Phase III clinical study of celecoxib-tramadol co crystal for pain management [5]. Based on increasing patents and reported research in this area, it can be expected that many new products will enter clinical pipeline and market in the near future. Furthermore, considering the fact that co crystals are not considered as new chemical entities by regulatory bodies, these can be explored as an innovative avenue for intellectual property scope broadening, non-patent infringing approach for ANDA products, 505 (b)(2) products, superior forms of challenging old drugs etc. In view of this, co crystal engineering research appears to be alluring especially in the industrial scenario to gain regulatory advantage and commercial benefit.

\section{References}

1. Kumar S, Nanda A (2017) Pharmaceutical Cocrystals: An Overview. Indian J Pharm Sci 79(6): 858-871.

2. Desai PP, Patravale VB (2017) Curcumin Cocrystal MicellesMultifunctional Nanocomposites for Management of Neurodegenerative Ailments. J Pharm Sci S0022-3549(17): 30836-30835.

3. Regulatory Classification of Pharmaceutical Co-Crystals Guidance for Industry.

4. http://www.ema.europa.eu/docs/en_GB/document_library/Scientific_ guideline/2015/07/WC500189927.pdf

5. Co-crystal E-58425 vs Tramadol and Celecoxib for Moderate to Severe Acute Pain After Bunionectomy. Phase III Clinical Trial.

\section{(c) () \\ This work is licensed under Creative} Commons Attribution 4.0 License

Submission Link: https://biomedres.us/submit-manuscript.php

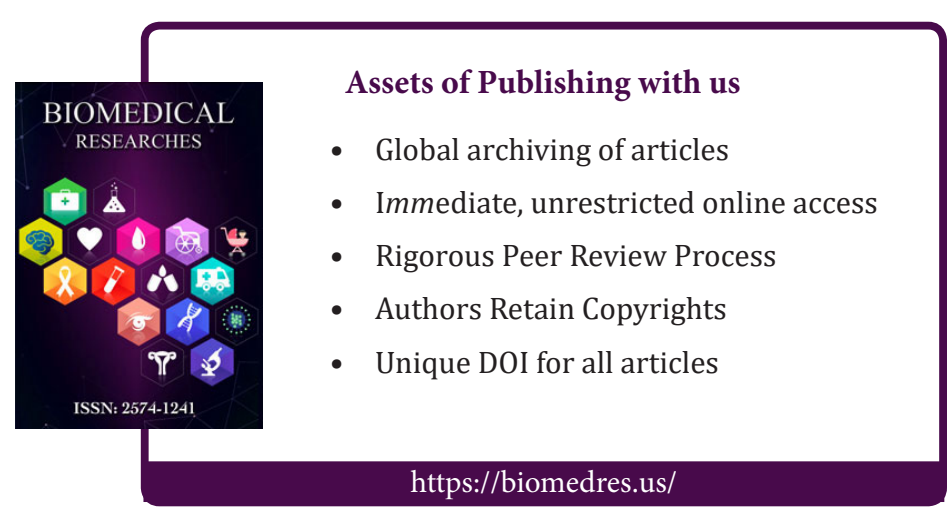

